\title{
Testes de mobilidade funcional são preditores de risco de queda em idosos praticante de atividade física?
}

http://dx.doi.org/10.11606/1807-5509201900030363

\section{Resumo}

0 objetivo do estudo foi analisar a validade de critério dos testes de mobilidade funcional para detectar o risco de queda em idosos. Participaram 557 idosos; destes, 180 idosos não praticantes de atividade fisica supervisionada (AFS) e 377 praticantes de AFS. Foram aplicados os testes timed up and go; timed up and go cognitive; timed up and go manual; teste apoio unipodal; alcance funcional; 5 repetitive chair test; step test; floor transfer; $360^{\circ}$ turn ; four square step test; e ordered multi-stepping over hoop. Os testes apresentaram capacidade discriminativa entre idosos caidores e não caidores na amostra geral e no grupo de idosos não praticantes de AFS. No entanto, os testes apresentaram baixa acurácia, sensibilidade e especificidade na amostra geral e boa sensibilidade e baixa especificidade nos idosos não praticantes de AFS. Os resultados sugerem que os instrumentos devem ser adequadamente testados antes de ser utilizados em ambientes ou perfil amostral de idosos.

PalavRas-chave: Instrumentação; Validade de Critério; Acurácia; Sensibilidade; Especificidade.

\section{Introdução}

Embora a diminuição da capacidade do equilíbrio postural seja um importante fator de quedas na população idosa, revisões sistemáticas têm sido limitadas em recomendar avaliação clínica subjetiva e o uso de testes clínicos tradicionais de mobilidade funcional para detectar o risco de queda $^{1,2}$. No entanto, há estudos que apontam vários instrumentos como supostamente preditores de risco de queda em idosos ${ }^{3-5}$, mas poucos estudos epidemiológicos têm-se centrado a priori sobre o déficit de controle postural como um dos fatores determinantes para a população idosa residente na comunidade.

O controle postural é um processo complexo que envolve a rápida integração automática de informaçôes a partir das dimensôes vestibular, sensorial, visual e musculoesquelética dos sistemas, na presença de cognição, e inclui a reação de tempo de atenção $0^{6-8}$. Os instrumentos de medição utilizados para avaliar o controle postural na prática clínica são um meio de quantificar a capacidade de interação dos componentes que permitem a estabilidade postural. O objetivo da triagem é identificar idosos que estão em risco elevado de sofrer queda e que devem receber uma avaliação mais aprofundada. $\mathrm{O}$ rastreio deve ter instrumento de medição simples e de fácil administração para que se possam identificar deficiências associadas ao risco de queda.

Vários estudos sustentam o uso de intrumentos (timed up and go; timed up and go cognitive; timed up and go manual; teste apoio unipodal; alcance funcional; 5 repetitive chair test; step test; floor transfer; $360^{\circ}$ turn ; four square step test; e ordered multi-stepping over hoop) que predizem o risco de queda em idosos residentes em comunidade, mas não fornecem medidas de validade ou confiabilidade para apoiar essas recomendaçóes ${ }^{1,9-16}$. A generalização dos resultados é ainda limitada pelos estudos de validação que visam a subpopulaçôes ${ }^{11-16}$; esse viés prejudica a validade científica do instrumento por não ser
*Centro de Ciências da Saúde e do Esporte, Universidade do Estado de Santa Catarina, Florianópolis, SC, Brasil. **Universidade do Oeste de Santa Catarina, Chapeco, SC, Brasil. 
analisada em uma amostra representativa. Do mesmo modo, é de amplo conhecimento que o desempenho em qualquer instrumento varia quando usado em diferentes contextos, podendo ser influenciado nas diferenças dos sujeitos, individuais e características ambientais ${ }^{17}$. Por conseguinte, outros estudos referem que esses testes não apresentam sensibilidade na detecção do risco de quedas em idosos residentes em comunidade, portanto, não se tem ainda clareza para qual perfil amostral esses testes são mais adequados para detectar o risco de queda. Adicionalmente, os testes clínicos de mobilidade funcional para detectar déficit de controle postural de idosos na sua maioria foram desenvolvidos para idosos institucionalizados e/ou com baixa condição física e com dificuldades para realizar suas atividades de vida diária (AVD), comprometendo sua validação científica ${ }^{12,15-19}$.

Ao analisar o desempenho dos vários instrumentos sob as mesmas condiçôes no mesmo ambiente, este estudo tentou identificar as diferenças na eficácia desses instrumentos na sua capacidade de discriminação e capacidade prognóstica no risco de queda, isto é, se há diferença na sua validade de critério em idosos praticantes e não praticantes de atividade física supervisionada (AFS). Embora não haja considerável sobreposição entre as características que compilam dos diversos instrumentos, este estudo apresenta a hipótese de que existem diferenças significativas no seu desempenho, principalmente ao comparar sua validade de critério em idosos praticantes e não praticantes de AFS. Uma vez que os idosos praticantes de AFS podem apresentar melhores níveis de aptidão física, isto é, capacidade funcional, os testes de mobilidade funcional podem possuir parâmetros de capacidade discriminativa e preditiva distintos para detectar o risco de queda em idosos praticantes e não praticantes de AFS, pois estudos apontam que a prática de atividade física regular e sistematizada ou de exercícios físicos regulares é capaz de minimizar os efeitos deletérios do envelhecimento, como a redução na massa muscular, colaborando para a manutenção da capacidade física (força e resistência muscular, equilíbrio e flexibilidade), capacidade aeróbica e autonomia do idoso ${ }^{20-22}$.

Estudos prévios ${ }^{23-26}$ relatam alta incidência de queda em idosos praticantes de AFS. Considera-se que os programas de exercícios físicos para idosos têm a finalidade de ser iniciados com avaliaçôes que permitem diagnosticar o mais precisamente possível as condiçôes reais que os idosos apresentam ao iniciá-los, como também a sua pré-disposição a sofrer quedas. Diante disso, tem-se a necessidade de esclarecer se os testes clínicos de mobilidade funcional são eficazes em predizer o risco de queda em idosos praticantes e não praticantes de AFS, uma vez que na literatura não se encontram estudos que estimaram a validação dos testes de mobilidade funcional em idosos praticantes de AFS, que se supóe idosos com alta capacidade funcional. $\mathrm{O}$ objetivo do estudo foi analisar a validade de critério (preditiva, discriminativa, convergente) dos testes de mobilidade funcional em idosos praticantes e não praticantes de AFS.

\section{Método}

Este estudo foi do tipo descritivo observacional transversal e utilizou a amostragem não probabilística por julgamento. Foi aprovado pelo comitê de ética da universidade local, por meio da plataforma Brasil, CAAE no 46613115.1.0000.0118, de acordo com a resolução no 466/2012 do National Health Council. Todos os participantes assinaram um termo de consentimento livre e esclarecido, incluindo os benefícios e riscos do estudo, que também foram previamente explicados.

\section{Amostra}

Participaram desta pesquisa 557 idosos residentes nas cidades de Chapecó e Florianópolis, em Santa Catarina, Brasil, com idade entre 60 e 94 anos (70,5 $\pm 6,1)$. Destes, $443(79,5 \%)$ não sofreram quedas nos últimos 12 meses e 114 (20,5\%) sofreram quedas. Dos idosos, $377(67,7 \%)$ praticavam regularmente atividade física supervisionada e $180(32,3 \%)$ não praticavam. Foram considerados praticantes de AFS os idosos que estavam matriculados há mais de seis meses nos programas de exercícios físicos, dentro das modalidades praticadas: hidroginástica $(10,3 \%)$, pilates $(8,8 \%)$, musculação $(16,7 \%)$, caminhada $(23,9 \%)$, ginástica $(7,2 \%)$ aula de dança $(3,7 \%)$, ioga $(6,9 \%)$, natação $(12,6 \%)$; basquetebol $(1,3 \%)$; ciclismo $(1,6 \%)$, corrida $(1,3 \%)$ e outros $(4,0 \%)$. 
Destes, $15,4 \%$ praticavam uma modalidade; $25,6 \%$ duas modalidades; $13,7 \%$ três modalidades; e $13 \%$ mais do que quatro modalidades de programas de exercícios físicos.

O estudo excluiu voluntários que apresentaram as seguintes ocorrências: histórico de cirurgia de membros inferiores e/ou coluna, nos últimos 12 meses; quadro álgico ou distúrbios musculoesqueléticos que impediram a manutenção da posição ortostática pelo tempo determinado no estudo (aproximadamente 30 minutos); déficit cognitivo pelo mini mental state exame - $\mathrm{MMSE}^{27}$, histórico de tratamento prévio para melhora do equilíbrio (participaram de intervenções que tinham objetivo de prevenir a queda, tais como reabilitação do sistema vestibular); distúrbios neurológicos na anamnese dos dados clínicos; e distúrbios no sistema cerebelar (teste de Romberg e do dedo-nariz).

\section{Procedimentos}

Inicialmente, os idosos foram encaminhados para um local silencioso, onde foi aplicada a ficha de anamnese e os questionários: Falls Efficacy Scale International (FES-I); Miniexame Do Estado Mental (MEEM); e Questionário de Atividade Física Habitual Baecke Modificado para Idoso (QBMI).

Após a aplicação dos questionários os idosos foram submetidos aos testes clínicos de mobilidade funcional, os quais foram executados de forma aleatória por meio de sorteio, com intervalo de descanso entre os testes entre 60 a 120 segundos: timed up and go-TUG ${ }^{16}$; timed up and go cognitive - TUG cognitive ${ }^{15}$; timed up and go manual - TUG manual $^{15}$; teste apoio unipodal ${ }^{28}$; alcance funcional $^{19}$; 5 repetitive chair test ${ }^{29}$; step test (ambos os membros inferiores $)^{12}$; floor transfer ${ }^{12}$; $360^{\circ}$ turn $^{18}$; four square step test-FSST ${ }^{18}$; ordered multi-stepping over hoop$O M O^{13}$. Os avaliadores instruíram e demonstraram a forma de execução dos testes aos idosos; todos os idosos realizaram uma tentativa de familiarização nos testes, com a finalidade de averiguar se tinham entendido a forma de execução deles.

\section{Instrumentação}

FALLS EFFICACY SCALE INTERNATIONAL - FES-I

A FES-I apresenta questões sobre a preocupação da possibilidade de cair ao realizar 16 atividades diferentes. Apresenta quatro possibilidades de respostas, com respectivos escores de 1 a 4 . O escore total pode variar de 16 a 64 , no qual o valor 16 corresponde à ausência de preocupação e 64 é preocupação extrema em relação às quedas durante a realização das atividades específicas do questionário. A respeito das categorias de resposta, a palavra "preocupado" expressa um desconforto racional ou cognitivo sobre a possibilidade de cair. Mas, ela não expressa o sofrimento emocional que poderia ser refletido por termos tais como "aflito", "ansioso" ou "medroso". A importância de se usar um termo não emocional se deve à possibilidade de os respondentes não quererem admitir emoçóes, o que poderia ser visto como sinal de fraqueza. A pobre autoeficácia caracterizada como "medo" pode ser fator pobre de comportamento, já que o "medo" apresenta conotação psiquiátrica que implica analogicamente a fobia ${ }^{30}$.

\section{MINIEXAME DO ESTADO MENTAL - MEEM}

O MEEM é um instrumento utilizado para rastreamento inicial do estado mental, que avalia a presença de déficit cognitivo, envolvendo duas categorias de respostas, verbais e não verbais. $\mathrm{O}$ MEEM é composto por 30 questóes categóricas, com uma escala de variação entre 0 a 30 pontos. A pontuação do teste classifica o déficit cognitivo em: 30 a 26, o indivíduo apresenta funçóes cognitivas preservadas; de 26 a 24, existe alteração não sugestiva de déficit; e 23 pontos ou menos, sugestão de déficit cognitivo. Os subtestes verbais medem, em particular, a orientação espaço-temporal, a memória imediata, a evocação e a memória de procedimento, a atenção e a linguagem. Os subtestes não verbais medem a coordenação perceptivo-motora e a compreensão de instruçóes ${ }^{31}$. A tradução do questionário MEEM foi realizada por LOURENÇO E VERAS ${ }^{32}$.

\section{QUESTIONÁRIO DE ATIVIDADE FÍSICA HABITUAL BAECKE MODIFICADO PARA IDOSO (QBMI)}

O questionário de atividade física habitual modificado por Baecke para idosos (QBMI) investiga a atividade física dos últimos 12 meses em idosos. Esse instrumento é composto por 16 questóes e abrange três componentes da atividade física: 1) atividades físicas ocupacionais; 2) exercícios físicos praticados durante o tempo de lazer; e 3) atividades físicas durante o tempo de lazer e atividades físicas de locomoção, excluindo exercícios físicos ${ }^{33}$. A tradução do questionário QBMI foi realizada por SIMÕEs ${ }^{34}$. 
TESTES CLÍNICOS DE MOBILIDADE FUNCIONAL

Para avaliar a dimensão de mobilidade do controle postural foi selecionado o teste timed up and go $-T U G^{16}$; para a dupla tarefa foram selecionados os testes timed up and go cognitive e timed up and go manual ${ }^{15}$; para o equilíbrio estático foi o teste apoio unipodal ${ }^{28}$; para o deslocamento do centro de gravidade foi o teste alcance funcional ${ }^{19}$; para a resistência muscular dos membros inferiores foram os seguintes testes: 5 repetitive chair test ${ }^{29}$, step test (ambos os membros inferiores) ${ }^{12}$ e floor transfer $^{12}$; para o sistema vestibular foi o $360^{\circ}$ turn $^{18}$; e, por fim, para a agilidade foi of four square step test $-F S S T^{18} \mathrm{e}$ o order ed multi-stepping over hoop-OMO ${ }^{13}$.

No teste TUG, o idoso iniciou na posição sentada, com as costas apoiadas na cadeira e seus antebraços descansados nos braço dela, sendo instruído a se levantar, andar um percurso linear de 3 metros até um ponto predeterminado marcado no chão, girar e tornar a sentar-se apoiando as costas na mesma cadeira, em linha reta; o tempo de execuçáo do teste foi registrado ${ }^{16}$.

$\mathrm{O}$ teste timed up and go manual (TUG manual) foi realizado nos mesmos procedimentos do $T U G$, porém, foi acrescentada a tarefa de carregar um copo de água durante a execução da trajetória do teste; o tempo de execução foi registrado ${ }^{15}$.

$\mathrm{O}$ teste timed up and go cognitive (TUG cognitive) também foi realizado nos mesmos procedimentos do $T U G$, acrescentando-se a tarefa cognitiva durante a sua execução; foi solicitado ao idoso que falasse nomes de animais durante a execução do teste; $o$ tempo de execução foi registrado ${ }^{15}$.

No teste apoio unipodal foi orientado que o idoso olhasse para um ponto fixo, colocando os braços ao lado do corpo e elevando uma das pernas (escolhida pelo próprio idoso), flexionando o joelho por 30 segundos. Não foi permitido que o idoso encostasse as pernas entre $\mathrm{si}^{28}$.

No teste alcance funcional, uma fita métrica foi presa à parede, paralela ao cháo, e posicionada na altura do acrômio do idoso, o qual foi posicionado com os pés confortáveis e paralelos entre si e separados na largura do quadril, perpendicularmente em relaçáo à parede e próximo ao início da fita métrica. $\mathrm{O}$ idoso foi instruído para elevar os membros superiores, com punho em posição neutra, cotovelo estendido e ombro com flexão de $90^{\circ}$. Após isso foi solicitado que o idoso, sem movimentar os pés, realizasse uma inclinação do tronco para frente, sem tocar a fita, o mais longe possível, sem perder o equilíbrio. Em seguida, foi verificar o deslocamento do idoso sobre a fita métrica ${ }^{19}$.

No 5 repetitive chair test foi orientado que o idoso se levantasse da cadeira com os braços cruzados sobre o peito e com as costas contra a cadeira cinco vezes, o mais rápido possível; o tempo de execução foi registrado ${ }^{29}$.

O step test avalia a capacidade do idoso em colocar um pé a $10 \mathrm{~cm}$ de altura e depois voltar para o chão repetidamente o mais rápido possível. A pontuação é o número de etapas concluídas no período de 15 segundos para cada membro inferior ${ }^{12}$.

No floor transfer o idoso foi orientado a sair da posição em pé e sentar-se ao chão sobre um colchonete e se levantar o mais rápido possível, o tempo de execução foi registrado ${ }^{12}$.

No teste $360^{\circ}$ turn foi solicitado ao idoso girar em torno do seu eixo em uma amplitude de $360^{\circ}$ nos sentidos horário e anti-horário, sem período de descanso; o tempo de execução foi registrado ${ }^{18}$.

No four square step test (FSST) o idoso foi posicionado em um quadro subdividido em quatro quadrantes numerados de 1 a 4 . No início do teste o idoso foi orientado a se posicionar no quadrante 1, atingindo os quadrantes 2,3 e 4; com um passo à frente, para trás e para os lados direito e esquerdo, seguindo exatamente essa sequência. $\mathrm{O}$ objetivo foi dar um passo táo rápido quanto possível em cada quadrante na seguinte sequência 2, 3, 4, 1, 4, 3, 2 e 1; o tempo de execução foi registrado ${ }^{12}$.

$\mathrm{O}$ ordered multi-stepping over hoop (OMO) é composto por um aro (raio $=0,7 \mathrm{~m}$ ) e um semicírculo concêntrico feito por fita (raio $=1,7$ $\mathrm{m}$, diâmetro do tubo $=0,03 \mathrm{~m})$ colocados no chão. $\mathrm{O}$ semicírculo concêntrico é dividido em três compartimentos e um quadrado numérico (números de 1 a 3; largura, $22 \mathrm{~cm}$; altura, $35 \mathrm{~cm}$ ) é colocado em cada compartimento. Há X marcas em cada seção, situadas a uma distância de $1 \mathrm{~m}$ do centro do aro. O idoso foi posicionado em pé no aro e foi orientado a entrar em cada compartimento e voltar para o aro, alternadamente, em ordem sequencial. O tempo de execução foi registrado. Todos os testes foram aplicados de acordo com o protocolo dos seus respectivos autores originais ${ }^{13}$.

\section{Estatística}

No tratamento estatístico foram utilizadas assimetria e curtose da curva da normalidade e estatística descritiva (média, desvio-padrão, 
porcentagem, frequência). O teste KolmogorovSmirnov indicou que as variáveis escalares não apresentaram distribuição normal. $\mathrm{O}$ teste $\mathrm{U}$ de Mann Whitney foi utilizado para comparação entre os grupos caidores e não caidores. $\mathrm{O}$ teste Q-quadrado foi utilizado para analisar as variáveis qualitativas sociodemográficas, incidência de queda e estado de saúde entre os grupos. A correlação de Spearman foi aplicada aos testes clínicos de mobilidade funcional entre si para averiguar a validade de convergência dos testes e se eles avaliam dimensões do mesmo constructo.
Para a análise da correlação de Spearman entre as variáveis adotaram-se os seguintes critérios: fraca magnitude, valores inferiores a 0,29; moderada magnitude, valores entre 0,30 e 0,69 ; forte magnitude, valores entre 0,70 e 0,99 ; e perfeita magnitude, valores em $1,00^{35}$. Todos os testes adotaram o nível de significância de $5 \%(\alpha=0,05)$. Foi utilizado o pacote SPSS 20.0 para as análises. Para análise da sensibilidade dos testes clínicos de mobilidade funcional, com a finalidade de detectar o risco de queda em idosos, foi utilizada a análise da curva ROC.

\section{Resultados}

Os grupos apresentaram homogeneidade com relação à idade, dados sociodemográficos e condiçáo de saúde (TABELA 1). A incidência de queda na amostra em geral foi de 20,5\%; ao se separar a amostra de acordo com a prática de atividade física, a amostra dos não praticantes de AFS apresentou incidência de $24,4 \%$ e para os praticantes de atividade física regular de $18,6 \%$, não expondo diferença significativa entre as amostras $(\mathrm{p}=0,86)$. Os idosos praticantes de AFS tiveram idade média de 70,35 $\pm 6,3$ anos e os não praticantes de AFS tiveram idade média de 74,64 \pm 8,0 anos.

TABELA 1 -Características sociodemográficas e condição de saúde dos idosos caidores e não caidores

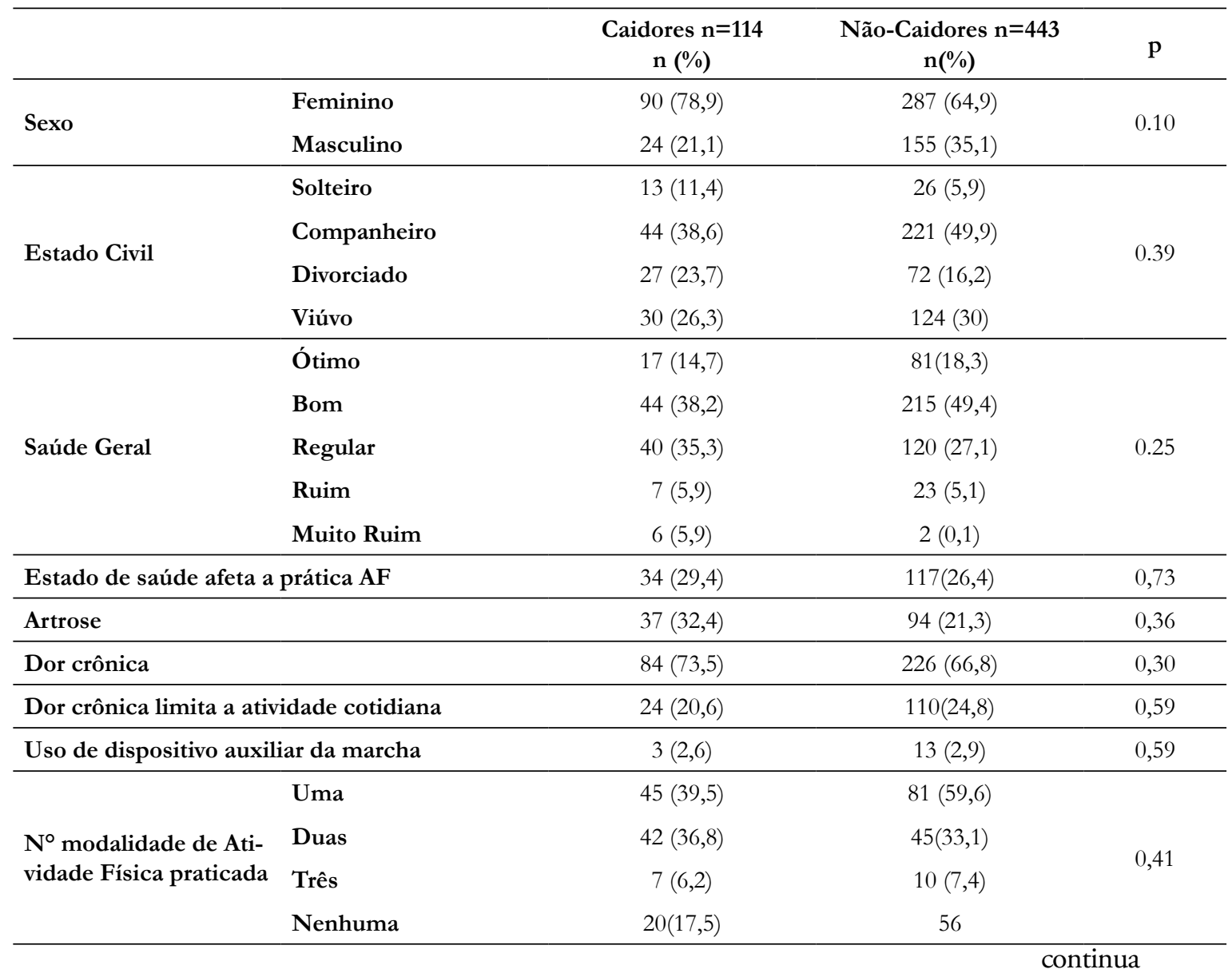


continuação

\begin{tabular}{lccc}
\hline & Média (DP) & Média (DP) & p \\
\hline Nível de Atividade Física (BQM) & $4,4 \pm 0,4$ & $5,1 \pm 0,2$ & 0,21 \\
\hline Medo de Queda (FES-I) & $32,9 \pm 3,0$ & $29,1 \pm 0,8$ & 0,67 \\
\hline Estado Cognitivo & $25,5 \pm 0,5$ & $24,5 \pm 0,3$ & 0,67 \\
\hline Idade & $71,1 \pm 7,4$ & $70,3 \pm 6,8$ & 0,45 \\
\hline
\end{tabular}

Ao analisar o desempenho dos testes clínicos de mobilidade funcional, os testes TUG, TUG cognitive, TUG manual, apoio unipodal, 5 repetitive chair test, tandem stand, alcance funcional, step test esquerdo e direito, floor transfer e o $360^{\circ}$ turn apresentaram capacidade discriminativa entre os idosos caidores e os não caidores $(\mathrm{p}<0,05)$. Os testes clínicos FSST e $O M O$ não apresentaram capacidade discriminativa
(TABELA 2). Ao analisar os testes de acordo com a atividade física, nos participantes do estudo que eram praticantes de AFS os testes de mobilidade funcional não expressaram capacidade discriminativa entre idosos caidores e não caidores (TABELA 3). Contudo, ao analisar o grupo dos não praticantes de AFS, os testes manifestaram capacidade discriminativa, exceto para os testes FSST e o OMO (TABELA 4).

TABELA 2 -Comparação do desempenho físico nos testes de mobilidade funcional entre os caidores e não caidores, praticantes de atividade física supervisionada.

\begin{tabular}{|c|c|c|c|c|}
\hline & & & & \\
\hline $\begin{array}{l}\text { timed up and go- } \\
T U G\end{array}$ & Teste & $\begin{array}{l}\text { Caidores ( } \mathrm{n}=114) \\
\text { Média (DP) }\end{array}$ & $\begin{array}{c}\text { Não Caidores }(\mathrm{n}=443) \\
\text { Média (DP) }\end{array}$ & $\mathrm{p}$ \\
\hline$O M O$ - ordered & TUG (s) & $10,0 \pm 3,3$ & $8,9 \pm 2,2$ & $<0,01 *$ \\
\hline multi-stepping over & TUG manual (s) & $10,07 \pm 3,9$ & $9,5 \pm 2,6$ & $<0,01 *$ \\
\hline s-segundos; & TUG cognitive (s) & $11,9 \pm 5,7$ & $10,6,0 \pm 4,3$ & $<0,01 *$ \\
\hline $\mathrm{r}-$ número & Apoio Unipodal(s) & $12,5 \pm 11,2$ & $15,4 \pm 11,8$ & $0,01 *$ \\
\hline de repetiçôes; & Alcance Funcional (cm) & $23,7 \pm 12,3$ & $26,9 \pm 9,8$ & $<0,01 *$ \\
\hline *diferença & 5 Repetitive Chair Test (r) & $13,7 \pm 4,4$ & $13,0 \pm 3,5$ & $0,04 *$ \\
\hline Significativa entre & Step Test Direito (r) & $13,9 \pm 5,7$ & $14,1 \pm 3,5$ & $0,03^{*}$ \\
\hline & Step Test Esquerdo (r) & $14,0 \pm 5,0$ & $14,4 \pm 3,6$ & $0,01 *$ \\
\hline & Floor Transfor (s) & $10,7 \pm 9,8$ & $8,3 \pm 4,6$ & $<0,01 *$ \\
\hline & $360^{\circ}$ Turn (s) & $86,7 \pm 3,0$ & $5,9 \pm 2,2$ & $<0,01 *$ \\
\hline & Four Square Step Test (s) & $14,6 \pm 7,7$ & $12,7 \pm 4,4$ & 0,13 \\
\hline & OMO (s) & $12,5 \pm 7,8$ & $11,7 \pm 5,3$ & 0,27 \\
\hline
\end{tabular}

AFS -

atividade física supervisionada; timed up and go$T U G$;

OMO-ordered multi-stepping over hoop;

s - segundos;

$\mathrm{r}$ - número

de repetiçóes;

*diferença

significativa entre os grupos $\mathrm{p}<0,05$.
TABELA 3 -Comparação do desempenho físico nos testes de mobilidade funcional entre os caidores e não caidores, praticantes de atividade física supervisionada.

\begin{tabular}{lcc}
\hline & \multicolumn{2}{c}{ Praticantes de AFS } \\
\cline { 2 - 3 } Teste & $\begin{array}{c}\text { Caidores (n=114) } \\
\text { Média (DP) }\end{array}$ & $\begin{array}{c}\text { Não Caidores (n=443) } \\
\text { Média (DP) }\end{array}$ \\
\hline TUG (s) & $9,1 \pm 0,4$ & $8,7 \pm 0,2$ \\
TUG manual (s) & $9,1 \pm 0,04$ & $9,2 \pm 0,2$ \\
TUG cognitive (s) & $11,9 \pm 1,1$ & $10,0 \pm 0,3$ \\
Apoio Unipodal(s) & $16,1 \pm 2,4$ & $15,7 \pm 1,0$ \\
Alcance Funcional (cm) & $26,0 \pm 5,9$ & $30,8 \pm 3,7$ \\
$\mathbf{5}$ Repetitive Chair Test (r) & $13,9 \pm 0,8$ & $12,5 \pm 0,3$ \\
\hline
\end{tabular}


continuação

\begin{tabular}{lccc}
\hline & \multicolumn{2}{c}{ Praticantes de AFS } & p \\
\cline { 2 - 3 } Teste & $\begin{array}{c}\text { Caidores (n=114) } \\
\text { Média (DP) }\end{array}$ & $\begin{array}{c}\text { Não Caidores (n=443) } \\
\text { Média (DP) }\end{array}$ & 0,56 \\
\hline Step Test Direito (r) & $14,1 \pm 0,9$ & $13,7 \pm 0,4$ & 0,66 \\
Step Test Esquerdo (r) & $14,2 \pm 0,9$ & $14,1 \pm 0,4$ & 0,40 \\
Floor Transfor (s) & $8,3 \pm 1,5$ & $7,0 \pm 0,5$ & 0,98 \\
$\mathbf{3 6 0}^{\circ}$ Turn (s) & $5,8 \pm 0,6$ & $6,0 \pm 0,2$ & 0,50 \\
Four Square Step Test (s) & $13,7 \pm 1,3$ & $13,1 \pm 0,5$ & 0,96 \\
OMO (s) & $11,8 \pm 1,7$ & $11,5 \pm 0,5$ & \\
\hline
\end{tabular}

TABELA 4 -Comparação do desempenho físico nos testes de mobilidade funcional entre os caidores e não caidores, não praticantes de atividade física supervisionada.

\begin{tabular}{lccc}
\hline \multirow{2}{*}{ Teste } & \multicolumn{2}{c}{ Não Praticantes de AFS } & p \\
\cline { 2 - 3 } & $\begin{array}{c}\text { Caidores (n=44) } \\
\text { Média (DP) }\end{array}$ & $\begin{array}{c}\text { Não Caidores (n=136) } \\
\text { Média (DP) }\end{array}$ & $<0,01^{*}$ \\
\hline TUG (s) & $11,1 \pm 3,2$ & $9,5 \pm 2,3$ & $<0,01 *$ \\
TUG manual (s) & $10,5 \pm 4,1$ & $10,3 \pm 2,8$ & $<0,01^{*}$ \\
TUG cognitive (s) & $12,3 \pm 5,9$ & $11,6 \pm 5,0$ & $<0,01 *$ \\
Apoio Unipodal(s) & $7,8 \pm 8,8$ & $13,0 \pm 11,6$ & $<0,01 *$ \\
Alcance Funcional (cm) & $20,3 \pm 8,6$ & $27,9 \pm 8,9$ & $0,04 *$ \\
$\mathbf{5}$ Repetitive Chair Test (r) & $15,0 \pm 4,3$ & $13,8 \pm 3,7$ & $<0,01^{*}$ \\
Step Test Direito (r) & $13,0 \pm 6,3$ & $14,1 \pm 3,3$ & $<0,01 *$ \\
Step Test Esquerdo (r) & $13,4 \pm 5,4$ & $14,2 \pm 3,4$ & $<0,01 *$ \\
Floor Transfor (s) & $10,6 \pm 6,4$ & $7,3 \pm 4,6$ & $<0,01 *$ \\
360 ${ }^{\circ}$ Turn (s) & $7,3 \pm 3,0$ & $6,1 \pm 2,5$ & 0,17 \\
Four Square Step Test (s) & $15,7 \pm 7,9$ & $13,0 \pm 4,6$ & 0,18 \\
OMO (s) & $13,2 \pm 7,0$ & $12,5 \pm 5,8$ & \\
\hline
\end{tabular}

AFS -

atividade física supervisionada; timed up and go $T U G$; OMO - ordered multi-stepping over hoop;

$s$ - segundos;

$\mathrm{r}$ - número

de repetiçōes;

*diferença

significativa entre os grupos $\mathrm{p}<0,05$.
Ao analisar a correlação entre os testes nota-se que todos apresentaram correlação significativa entre si, sendo que os testes alcance funcional e tandem stand exibiram muito fraca correlação com os outros testes; os testes floor transfer e apoio unipodal apresentaram fraca a moderada correlação com os outros testes. Os testes timed up and go, timed up and go cognitive, timed up and go manual, 5 repetitive chair test, step test, $360^{\circ}$ turn, four square step test e ordered multi-stepping over hoop revelaram moderada a forte correlação entre si (TABELA 5).

Ao analisar a curva ROC dos testes de mobilidade funcional nota-se que não houve teste que apresentasse bons níveis de sensibilidade e especialidade, exceto os testes FSST e OMO, na amostra geral (TABELA 6). Ao analisar a curva ROC dos testes na amostra de idosos não praticantes de AFS observa-se que os testes de mobilidade funcional apresentaram aumento da área da curva ROC, sendo que os testes TUG, TUG cognitive, apoio unipodal e alcance funcional apresentaram boa sensibilidade; os testes TUG manual, floor transfer, step test direito e esquerdo, $360^{\circ}$ turn e tandem stand manifestaram razoável sensibilidade; entretanto, os testes tiveram especificidade baixa, exceto o apoio unipodal e o TUG cognitive, que obteve especificidade razoável (TABELA 7). 
Moreira ACSS, et al.

TABELA 5-Correlação entre os testes clínicos de mobilidades funcional

\begin{tabular}{|c|c|c|c|c|c|c|c|c|c|c|c|c|}
\hline \multirow{3}{*}{$\begin{array}{l}\text { TUG - timed up } \\
\text { and go; } \\
\text { OMO - ordered } \\
\text { multi-stepping over }\end{array}$} & \multirow{2}{*}{\multicolumn{2}{|c|}{ FSST }} & \multirow[b]{2}{*}{$\begin{array}{l}360^{\circ} \\
\text { Turn }\end{array}$} & \multirow[b]{2}{*}{$\begin{array}{l}\text { Floor } \\
\text { Transfer }\end{array}$} & \multirow[b]{2}{*}{$O M O$} & \multirow[b]{2}{*}{$\begin{array}{c}\text { Step } \\
\text { Esquer- } \\
\text { do }\end{array}$} & \multirow[b]{2}{*}{$\begin{array}{c}\text { Step } \\
\text { Direito }\end{array}$} & \multirow[b]{2}{*}{$\begin{array}{c}\text { Alcance } \\
\text { Funcio- } \\
\text { nal }\end{array}$} & \multirow[b]{2}{*}{$\begin{array}{l}\text { 5-r } \\
\text { Chair }\end{array}$} & \multirow[b]{2}{*}{$\begin{array}{c}\text { Apoio } \\
\text { Unipo- } \\
\text { dal }\end{array}$} & \multirow[b]{2}{*}{$\begin{array}{l}\text { TUG } \\
\text { cognitive }\end{array}$} & \multirow[b]{2}{*}{$\begin{array}{l}\text { TUG } \\
\text { manua }\end{array}$} \\
\hline & & & & & & & & & & & & \\
\hline & $T U G$ & $0,51 *$ & $0,55^{*}$ & $0,36^{*}$ & $0,50^{*}$ & $-0,47^{*}$ & $-0,46^{*}$ & $-0,28^{*}$ & $0,58^{*}$ & $-0,39 *$ & $0,73^{*}$ & $0,85^{*}$ \\
\hline $\begin{array}{l}\text { hoop: } \\
5-r \text { chair }-5\end{array}$ & $\begin{array}{c}\text { TUG } \\
\text { Manual }\end{array}$ & $0,51 *$ & $0,51 *$ & $0,33^{*}$ & $0,46^{*}$ & $-0,39 *$ & $-0,38^{*}$ & $-0,23^{*}$ & $0,52 *$ & $-0,44 *$ & $0,723^{*}$ & \\
\hline $\begin{array}{l}\text { repetitive chair test; } \\
\text { FSST - four square }\end{array}$ & $\begin{array}{l}\text { TUG } \\
\text { Cognitive }\end{array}$ & $0,43^{*}$ & $0,48^{*}$ & $0,32^{*}$ & $0,44^{*}$ & $-0,45^{*}$ & $-0,43 *$ & $-0,27 *$ & $0,44^{*}$ & $-0,36 *$ & & \\
\hline \multirow{8}{*}{$\begin{array}{l}\text { step test; } \\
* \text { d i f e r e n ç a } \\
\text { significativa entre } \\
\text { os grupos } \mathrm{p}<0,001 \text {. }\end{array}$} & $\begin{array}{c}\text { Apoio } \\
\text { Unipo- } \\
\text { dal }\end{array}$ & $-0,30 *$ & $-0,29 *$ & $-0,22^{*}$ & $-0,32^{*}$ & $0,32^{*}$ & $0,30^{*}$ & $0,31 *$ & $-0,27 *$ & & & \\
\hline & $\begin{array}{l}\text { 5-r } \\
\text { Chair } \\
\text { Test }\end{array}$ & $0,45^{*}$ & $0,40^{*}$ & $0,24 *$ & $0,34 *$ & $-0,42^{*}$ & $-0,39 *$ & $-0,25^{*}$ & & & & \\
\hline & $\begin{array}{c}\text { Alcance } \\
\text { Funcio- } \\
\text { nal }\end{array}$ & $-0,29 *$ & $-0,21 *$ & $-0,18^{*}$ & $-0,32^{*}$ & $0,26^{*}$ & $0,26 *$ & & & & & \\
\hline & $\begin{array}{c}\text { Step } \\
\text { Direito }\end{array}$ & $-0,38 *$ & $-0,34 *$ & $-0,30 *$ & $-0,40^{*}$ & $0,89 *$ & & & & & & \\
\hline & $\begin{array}{l}\text { Step } \\
\text { Esquer- } \\
\text { do }\end{array}$ & $-0,42^{*}$ & $-0,36^{*}$ & $-0,32 *$ & $-0,40^{*}$ & & & & & & & \\
\hline & $O M O$ & $0,42 *$ & $0,43^{*}$ & $0,23^{*}$ & & & & & & & & \\
\hline & $\begin{array}{l}\text { Floor } \\
\text { Transfer }\end{array}$ & $0,32^{*}$ & $0,33^{*}$ & & & & & & & & & \\
\hline & $\begin{array}{l}360^{\circ} \\
\text { Turn }\end{array}$ & $0,48^{*}$ & & & & & & & & & & \\
\hline
\end{tabular}

TABELA 6-Análise da curva ROC, sensibilidade e especificidade dos testes de mobilidade funcional na amostra PC - ponto de corte; $S$ - sensibilidade; E - especialidade; ICC - intervalo de confiança de 95\%; p - nível de significância $(\mathrm{p}<0,05)$; TUG-timed up and go; OMO - ordered multi-stepping over hoop; s - segundos; $\mathrm{cm}$ - centímetros; $\mathrm{r}$ - número de repetiçôes; ns- não significativo. geral.

\begin{tabular}{|c|c|c|c|c|c|c|}
\hline Teste & PC & $E(\%)$ & $\mathrm{S}(\%)$ & Área & ICC & $\mathrm{p}$ \\
\hline TUG manual (s) & 10 & 52 & 40 & 0,58 & $0,48-0,68$ & $<0,01$ \\
\hline TUG cognitive (s) & 10 & 52 & 34 & 0,59 & $0,49-0,68$ & $<0,01$ \\
\hline Apoio Unipodal(s) & 6,5 & 52 & 37 & 0,59 & $0,52-0,66$ & 0,03 \\
\hline Step Test Direito (r) & 12 & 43 & 30 & 0,58 & $0,50-0,66$ & 0,03 \\
\hline Step Test Esquerdo (r) & 12 & 43 & 28 & 0,59 & $0,52-0,66$ & 0,01 \\
\hline OMO (s) & ns & ns & ns & 0,50 & $0,43-0,59$ & 0,27 \\
\hline
\end{tabular}


TABELA 7-Análise da curva ROC, sensibilidade e especificidade dos testes de mobilidade funcional na amostra de não praticantes de atividade física.

\begin{tabular}{lcccccc}
\hline Teste & PC & E (\%) & S (\%) & Área & ICC & p \\
\hline TUG (s) & 8,5 & $80 \%$ & $50 \%$ & 0,69 & $0,60-0,78$ & $<0,01$ \\
TUG manual (s) & 10.5 & $70 \%$ & $44 \%$ & 0,58 & $0,55-0,74$ & $<0,01$ \\
TUG cognitive (s) & 9.5 & $88 \%$ & $67 \%$ & 0,67 & $0,57-0,76$ & $<0,01$ \\
Apoio Unipodal(s) & 12.5 & $84 \%$ & $60 \%$ & 0,66 & $0,57-0,75$ & 0,02 \\
5 Repetitive Chair Test (r) & $\mathrm{ns}$ & $\mathrm{ns}$ & $\mathrm{ns}$ & 0,60 & $0,50-0,70$ & 0,05 \\
Alcance Funcional (cm) & 26,5 & $82 \%$ & $43 \%$ & 0,74 & $0,66-0,82$ & $<0,01$ \\
Step Test Direito (r) & 13.5 & $73 \%$ & $49 \%$ & 0,66 & $0,56-0,76$ & $<0,01$ \\
Step Test Esquerdo (r) & 14.5 & $75 \%$ & $53 \%$ & 0,63 & $0,54-0,73$ & $<0,01$ \\
OMO (s) & $\mathrm{ns}$ & $\mathrm{ns}$ & $\mathrm{ns}$ & 0,50 & $0,38-0,62$ & 0,98 \\
Floor Transfore (s) & 6.5 & $73 \%$ & $43 \%$ & 0,68 & $0,56-0,80$ & $<0,01$ \\
360 ${ }^{\circ}$ Turn (s) & 6.5 & $50 \%$ & $30 \%$ & 0,65 & $0,53-0,76$ & $<0,01$ \\
Four Square Step Test (s) & $\mathrm{ns}$ & $\mathrm{ns}$ & $\mathrm{ns}$ & 0,61 & $0,48-0,73$ & $<0,01$ \\
\hline
\end{tabular}

$\mathrm{PC}$ - ponto de corte;

$S$ - sensibilidade;

E - especialidade;

ICC - intervalo de

confiança de 95\%;

p - nível de signi-

ficância $(\mathrm{p}<0,05)$;

$T U G$-timed up and

go; OMO - ordered

multi-stepping over

hoop; s - segundos;

cm - centímetros;

r - número de re-

petições; ns - não significativo.

\section{Discussão}

$\mathrm{Na}$ literatura não se encontrou nenhum estudo que teve a finalidade de averiguar a validade dos instrumentos de mobilidade funcional para predizer o risco de queda em idosos praticantes de AFS. Os resultados comprovaram a hipótese deste estudo de que os idosos praticantes de AFS apresentam melhores condições de mobilidade funcional do que os idosos não praticantes de AFS. Logo, os testes de mobilidade funcional não apresentaram capacidade discriminativa e, consequentemente, preditiva em prognosticar caidores e não caidores em idosos praticante de AFS, mas, mantiveram sua capacidade $\mathrm{d}$-iscriminativa e preditiva para idosos não praticantes de AFS.

$\mathrm{Na}$ literatura existem diversos testes de mobilidade funcional com alguma evidência para apoiar a sua utilização na predição de risco de quedas em idosos residente em comunidade ${ }^{1,9-16}$. A escolha de um teste num contexto clínico necessita refletir a finalidade para a qual ele será destinado. Se o objetivo do estudo for executar uma triagem de populações de alto risco de queda é necessário um instrumento rápido e de fácil aplicação e ainda deve ter boa sensibilidade e especificidade. Se o objetivo for reduzir o risco de queda, o instrumento precisa identificar de forma viável fatores de risco remediáveis em que intervenções podem ser focadas. A seleção de um instrumento adequado é dificultada pela falta de consistência nos métodos de aplicação e interpretação das propriedades comparativas do instrumento na literatura publicada. Por exemplo, os critérios para determinar os valores preditivos elevados para instrumentos de avaliação de risco de queda são recomendados para a sensibilidade acima de $80 \%$ e especificidade acima de $75 \%{ }^{17,36}$.

Com base no valor preditivo recomendando pela literatura, mencionado previamente, os testes de mobilidade funcional, nos participantes deste estudo, apresentaram baixa sensibilidade (variando entre $43 \%$ e $58 \%$ ) e especificidade (variando entre $28 \%$ e $46 \%$ ), sendo que o $O M O$ e o FSST não apresentaram validade preditiva. No entanto, ao analisarmos em idosos não praticantes de AFS os testes TUG, TUG manual, TUG cognitive, apoio unipodal e alcance funcional revelaram sensibilidade adequada, porém, baixa especificidade. Os resultados indicam que os testes não são suficientes para ser utilizados como instrumentos de triagem simplificada para detectar o risco de queda. Esse achado vai ao encontro de estudos prévios que avaliaram os testes $T U G^{1,11,37-}$ 42 , apoio unipodal ${ }^{37,42,43}$, step test ${ }^{18}$ e alcance funcional ${ }^{43,44}$, que evidenciaram baixa validade preditiva dos testes em outros perfis amostrais da população idosa.

A baixa validade preditiva dos testes pode ser devido a vários fatores, ao considerar que o fenômeno quedas é multifatorial tornando improvável que se explicado usando um único teste. Os testes de mobilidade funcional medem indiretamente vários 
fatores da capacidade funcional associaciados ao risco de queda, incluindo força, resistência muscular, agilidade, tempo de reação, equilíbrio, coordenação motora, entre outros ${ }^{8,17,19,29,45,46}$, mas, podem não refletir suficientemente fatores de risco como condição de saúde, estado cognitivo, estado mental, efeito de medicamentos, fatores ambientais, entre outros. Sugere-se considerar a associação com outros fatores de risco de queda para fornecer informaçôes adicionais sobre a identificação dos idosos com potencial a sofrer quedas futuras.

A área da curva ROC (AUC) é outro parâmetro para analisar a validade preditiva do intrumento. Neste estudo a AUC dos testes foi baixa na amostra em geral e no grupo de idosos náo praticantes de AFS, sendo considerados pela literatura valores da AUC próximos de 0,50 indicando que o teste náo apresenta poder discriminante, sendo recomendado que a AUC tenha valor igual ou superior a 0,70 , sugerindo que o teste é satisfatório para predizer a ocorrência do fenômeno ${ }^{47}$. Os testes neste estudo apresentaram AUC com valores entre 0,50 e 0,62 na amostra geral; sugere-se comprometimento da sua capacidade discriminativa e preditiva. Mas, ao analisar os idosos não praticantes de AFS, notou-se melhora da AUC dos testes (exceto em 5 repetitive chair test, OMO e FSST), apresentando valores entre 0,60 a 0,74 , sugerindo que os testes apresentam maior poder discriminativo nesse perfil amostral, no entanto, ainda não satisfatório para utilizá-los como testes de triagem, sendo necessária a associação de outros fatores para investigar o risco de queda nesse perfil amostral de idosos.

O baixo valor da AUC apresentado nos testes, neste estudo, pode ser explicado, principalmente, pela baixa especificidade apresentada por eles, indicando uma importante atenuação do poder discrimanativo dos testes em identificar idosos não caidores na nossa amostra. De acordo com VASSALLO et al. ${ }^{47}$, a baixa especificidade de um instrumento é esperada em um ambiente no qual os idosos são impedidos de cair, com alta sensibilidade. Esse fenômeno ocorreu com os resultados do teste alcance funcional, em idosos não praticantes de AFS, reduzindo a sua AUC, o que era também esperado que ocorresse nos idosos praticantes de AFS. A alta sensibilidade e baixa especificidade sugerem que outros fatores interferem para que os idosos não sofram quedas, havendo necessidade de estudos para investigar quais são os fatores mediadores que atuam para a redução da incidência de queda nesse perfil amostral e se são eficazes ao longo do tempo.
No entanto, os testes nos idosos praticantes de AFS apresentaram baixa AUC, próxima a 50\%, com baixa sensibilidade e especificidade, evidenciando incapacidade discriminativa e preditiva para esse perfil amostral, não sendo recomendada a aplicação dos testes, analisados por este estudo, em idosos praticantes de AFS, nos critérios de mensuração quantitativa.

O instrumento clínico ideal, com alta sensibilidade e especificidade, é difícil de desenvolver e a medida mais importante de um instrumento de avaliação de risco de queda é sem dúvida a sua sensibilidade. Uma forma possível de melhorar a avaliação de risco de queda é a concentraçaó em estratégias motoras existentes nos testes acrescentando meios de aumentar o desafio do controle postural para executá-los, assim, sua sensibilidade pode melhorar. $\mathrm{Na}$ visão de VASSALlo et al. ${ }^{47}$, desenvolver novos instrumentos para obter uma alta AUC, com alta sensibilidade e especificidade em ambientes distintos da validação, é, portanto, um processo fútil e inútil. A perfeição da AUC sugere que os desempenhos dos instrumentos e do objetivo da sua utilização são uniformes em todos os ambientes clínicos, o que não é verdade, uma vez que cada ambiente tem a sua particularidade ${ }^{17,47-49}$.

A acurácia de um teste preditivo é mais bem determinada por comparação com um teste de referência padrão, considerado padrão ouro. Atualmente, na literatura, ainda não está esclarecido o teste de referência padrão para predizer queda em idosos, visto que é um fenômeno multicausal e que um único teste de mobilidade funcional é incapaz de avaliar todos os parâmetros do controle postural. Para que o pesquisador possa avaliar a utilidade do teste de acordo com sua realidade na prática clínica, isto é, apurar se o teste subestima ou superestima a capacidade de detectar o fenômeno na população, faz-se necessário conhecer que atributo do controle postural o instrumento está mensurando. A validação convergente permite averiguar os testes que avaliam atributos do mesmo construto relacionado; valores de " $r$ " acima de 0,509 são considerados como indicativo de que os instrumentos mensuram o mesmo atributo do constructo, o que caracteriza a nomenclatura de evidência de validade convergente. Índices de correlação baixos ou moderados, com valores de " $r$ " entre 0,20 e 0,50, sugerem que os testes apresentam atributos relacionados ao mesmo constructo, porém, distintos ${ }^{49}$. Os resultados indicaram que os testes TUG e TUG manual apresentaram alto 
nível de convergência com os testes $360^{\circ}$ turn e o 5 repetitive chair test, indicando que os testes mensuram o mesmo atributo do constructo, o que faz sentido, uma vez que as tarefas motoras desses dois testes estavam inseridas dentro das tarefas motoras do TUG e TUG manual. Um giro de $180^{\circ}$ e o levantar e sentar da cadeira são componentes da tarefa motora do TUG e do TUG manual, entáo, indivíduos com melhor capacidade de girar, levantar e sentar tenderão a ter melhor desempenho nos testes; os mesmos resultados foram evidenciados em estudos prévios $^{50,51}$. No entanto, o TUG cognitive apresentou nível moderado de correlação com o 5 repetitive chair test e $360^{\circ}$ turn, supóe-se que a dimensão de maior influência no desempenho do TUG cognitive a ser mensurada é próprio estado cognitivo, náo a execução da tarefa motora propriamente dita. Os outros testes apresentaram correlação fraca a moderada entre si, indicando que os testes mensuram atributos diferentes do mesmo constructo, sendo comprovado por outros estudos prévios $^{12,13,18,38,50,52,53}$.

Este estudo possui várias limitações. Primeiramente, é transversal, com histórico de queda retrospectiva de autorrelato, o que pode ter comprometido a validade preditiva e a capacidade discriminativa dos testes, principalmente em idosos praticantes de AFS, uma vez que foram baseados na suposição de que a função física não mudou após a ocorrência de queda e permaneceu relativamente inalterada durante $o$ ano anterior, o que náo é fato fidedigno, uma vez que após a queda é normal o idoso reduzir sua confiança ao executar suas atividades de vida diária (AVD). Assim, pressupóese que pode diminuir a sua capacidade funcional e não somente o medo da queda, mas também que com medo seja incapaz de se levantar uma vez que cair. A baixa autoconfiança em realizar as atividades do dia a dia, pelo medo de cair, tende a provocar um comprometimento progressivo da capacidade funcional ao longo do tempo. Estudos futuros devem utilizar desenho prospectivo para superar essa limitação. Segundo, o autorrelato pode ter gerado viés no estudo, uma vez que envolve a capacidade do idoso de se lembrar da ocorrência do fenômeno de queda, o qual pode ter sido esquecido, uma vez que envolve um período longo de 12 meses, isto é, sua capacidade temporal. Terceiro, os idosos praticantes de AFS são mais jovens do que os idosos não praticantes de AFS, o que pode ter influenciando na capacidade discriminativa e preditiva dos testes. E, por fim, este estudo não averigou a influência de outros fatores intrínsecos (estado de saúde, medicamentos, estado cognitivo, medo de cair, nivel da atividade física, entre outros) e ambientais nos testes de mobilidade funcional, o que pode ter influenciado os resultados deste estudo.

Os resultados deste estudo têm alta relevância clínica e acadêmica, pois sugerem que a seleção clínica de intrumento para predizer o risco queda em idosos deve se amparar em intrumentos que apresentam estudos da sua validade e confiabilidade de previsão para o perfil amostral de sua população a ser estudada. Instrumentos que apresentam apenas subcomponentes com validade preditiva não devem ser recomendado para uso até que sejam revistos e retestados, confirmando sua validade e confiabilidade para o perfil amostral que pretende estudar, uma vez que os resultados manisfestaram comportamentos distintos de acordo com a prática de AFS dos idosos, indicando que os testes de mobilidade funcional não apresentaram capacidade discriminativa e, consequentemente, validade pretidiva, indicando fragidade dos testes nesse perfil da amostra da população idosa. Nos idosos não praticantes de AFS os testes de mobilidade funcional apresentaram capacidade discriminativa, exceto FSST e OMO. Entretanto, os testes TUG, TUG manual, TUG cognitive, apoio unipodal e alcance funcional revelaram boa sensibilidade, porém, baixa especificidade, sugerindo que os testes não são suficientes para ser utilizados como um instrumento de triagem simplificada para detectar o risco de queda na amostra estudada, o nível de especificidade nos testes está sob o efeito de algum fator mediador que minimiza a incidenência de queda nesse perfil amostral, necessitando-se da realização de estudos que comprovem a influência de fatores intrísecos e extrínsecos de risco de queda para fornecer informaçóes adicionais sobre a identificação dos idosos com potencial a sofrer quedas futuras.

Os testes indicaram, pela análise da correlação, que avaliam distintos atributos do mesmo constructo. TUG, TUG cognitive e o TUG manual apresentaram correlação alta, sugerindo que avaliam o mesmo atributo do constructo, assim, não é sugerida a aplicação dos testes simutaneamente na população, sendo que somente um teste é suficiente para avaliar o referido atribuído. A necessidade de uma maior investigação da influência de fatores intrínsecos e extrínsecos no desempenho do teste com a finalidade de constatar a teoria dos fatores de risco de queda neste perfil amostral ainda não está bem esclarecida. 


\begin{abstract}
Functional mobility tests are predictors of fallrisk in elderly practice of physical activity?

The objective of the study was to analyze the validity of the criterion of functional mobility tests to detect the falls risk in the elderly. A total of 557 elderly, of these 180 non-supervised physical activity (SPA) and 377 SPA practitioners participated. The following tests were applied: timed up and go, timed up and go cognitive, timed up and go manual, one leg stand test, functional reach test, 5 repetitive chair test, step test, floor transfer, $360^{\circ}$ turn, four square step tests and ordered multi-stepping over hoop. The tests presented discriminative capacity between elderly falls and non-falls in the general sample and in the non-SPA elderly group. However, the tests presented low accuracy, sensitivity and specificity in the general sample, and good sensitivity and low specificity in the non-SPA elderly. The results suggest that the instruments should be adequately tested before being used in environments or the sample profile of the elderly.
\end{abstract}

KEYwords: Instrumentation; Validity the Criterion; Accuracy; Sensitivity; Specificity.

\title{
Referências
}

1. Schoene D,Wu SM, Mikolaizak AS, Menant JC, Smith ST, Delbaere K, et al. Discriminative ability and predictive validity of the timed up and go test in identifying older people who fall: systematic review and meta-analysis. J Am Geriatr Soc. 2013;61(2):202-8.

2. Barry E, Galvin R, Keogh C, Horgan F, Fahey T. Is the Timed Up and Go test a useful predictor of risk of falls in community dwelling older adults: a systematic review and meta-analysis. BMC Geriatr. 2014;1:1-14.

3. Reider NC, Naylor PJ, Gaul C. Sensitivity and specificity of the minimal chair height standing ability test: a simple and affordable fall-risk screening instrument. J Geriatr Phys Ther. 2015;38(2):90-5.

4. Agmon M, Armon G. A cross-sectional study of the association between mobility test performance and personality among older adults. BMC Geriatr. 2016;181816:105. doi: 10.1186/s12877-016-0272-8.

5. Yoo IY. Recurrent falls among community-dwelling older Koreans: prevalence and multivariate risk factors. J GerontolNurs. 2011;37(9):28-40.

6. Horak FB, Kluzik J, Hlavacka F. Velocity dependence of vestibular information for postural control on tilting surfaces. J Neurophysiol. 2016;1116(3):1468-79.

7. Alonso AC, Peterson MD, Busse AL, Jacob-Filho W, Borges MTA, Serra MM, et al. Muscle strength postural balance and cognition are associated with braking time during driving in older adults. Exp Gerontol. 2016; 185:13-17.

8. Horak FB. Postural orientation and equilibrium: what do we need to know about neural control of balance to prevent falls.Age Ageing. 2006; 35(S2):ii7-ii11.

9. Bonguea B, Duprea C, Beaucheta O, Rossata A, Fantino B, Colveza A. A screening tool with five risk factors was developed for fall-risk prediction in community-dwelling elderly. J Clin Epid. 2011;64:1152-60.

10. Haines T, Kuys SS, Morrison G, Clarke J, Bew P. Balance Impairment Not Predictive of Falls in Geriatric Rehabilitation Wards. J Gerontol A Biol Sci Med Sci. 2008;63(5):523-28.

11. Chiu AY, Au-Yeung SS, Lo SK. A comparison of four functional tests in discriminating fallers from non-fallers in older people. Disabil Rehabil. 2003;725(1):45-50.

12. Murphy MA, Olson SL, Protas EJ, Overby AR. Screening for falls in community-dwelling elderly. J Aging Phys Act. 2003;11:64-78.

13. Tsutsumimoto K, Doi T, Misu S, Ono R, Hirata S. Can the Ordered Multi-Stepping Over Hoop test be useful for predicting fallers among older people? A preliminary 1 year cohort study. Aging Clin Exp Res. 2013;25(4):427-432.

14. Wrisley DM, Kumar NA. Functional gait assessment: concurrent discriminative and predictive validity in communitydwelling older adults. Phys Ther. 2010;90(5):761-773.

15. Shumway-Cook A, Brauer S, Woollacott M. Predicting the probability for falls in community-dwelling older adults using the Timed Up \& Go Test. Phys Ther. 2000;80(9):896-903.

16. Podsiadlo D, Richardson S. The timed "Up \& Go": a test of basic functional mobility for frail elderly persons. J Am 
Geriatr Soc. 1991;39(2):142-148.

17. Berg K, Wood-Dauphinee SL, Williams JI, Maki B. Measuring balance in the elderly: Validation of an instrument. Can J Public Health. 1992;83(S2):S7-11.

18. Dite W, Temple VA. A clinical test of stepping and change of direction to identify multiple falling older adults.Arch Phys Med Rehabil. 2002;83(11):1566-71.

19. Duncan PW, Weiner DK, Chandler J, Studenski S. Functional reach: a new clinical measure of balance. J Gerontol. 1990;45(6):M192-M197.

20. Gomes NM, Castro MF de. Estudo comparativo da independência funcional e qualidade de vida entre idosos ativos e sedentários. Rev Bras Med Esporte. 2012;18(4):234-237.

21. Silva MF, Goulart NBA, Lanferdini FJ, Marcon M, Dias CP. Relação entre os níveis de atividade física e qualidade de vida de idosos sedentários e fisicamente ativos. Rev bras geriatr gerontol. 2012;15(4):634-642.

22. Gonçalves MM. Atividade física e funcionalidade do idoso. Motriz. 2010;16(4):1024-1032.

23. Santos GM, Souza ACS, Virtuoso JF, Tavares GMS, Mazo GZ. Predictive values at risk of falling in physically active and no active elderly with Berg Balance Scale. Rev Bras Fisioter. 2011;15(2):95-101.

24. Silva EC, Duarte NB, Arantes PMM. Estudo da relação entre o nível de atividade física e o risco de quedas em idosos. Fisioter Pesq. 2011;18(1):23-30.

25. Pimentel RM, Scheicher ME. Comparação do risco de queda em idosos sedentários e ativos por meio da escala de equilíbrio de Berg. Fisioter Pesq. 2009;16(1):6-10.

26. Mazo GZ, Liposcki DB, Ananda C, Prevê D. Condiçóes de saúde, incidência de quedas e nível de atividade física dos idosos. Rev bras fisioter. 2007;11(6):437-442.

27. Manubens JM, Martínez-Lage P, Martínez-Lage JM, et al. Variación de lãs puntuaciones em el Mini-Mental State com la edad y el nivel educativo. Datos normalizados em La población mayor de 70 años de Pamplona. Neurología. 1998;13:111-19.

28. Gabell A, Simons M. Balance coding. Physiotherapy. 1982;68:286-88.

29. Guralnik JM, Ferrucci L, Simonsick EM, Salive ME, Wallace RB. Lower-extremity function in persons over the age of 70 years as a predictor of subsequent disability. N Engl J Med.1995;332:556-561.

30. Yardley L, Beyer N, Hauer K, Kempen G, Piot-Ziegler C, Todd C. Development and initial validation of the falls efficacy scale-international (FES-I). Age Ageing. 2005;34(6):614-9.

31. Folstein MF, Folstein SE, McHugh PR. "Mini-mental state". A practical method for grading the cognitive state of patients for the clinician. J Psychiatr Res. 1975;12(3):189-98.

32. Lourenço RA, Veras RP. Mini-Mental State Examination: psychometric characteristics in elderly outpatients. Rev. Saude Publica. 2006;40(4):712-19.

33. Baecke JAH, Burema J, Frijters JER. A short questionnaire for the measurement of habitual physical activity in epidemiological studies. Am J Clin Nutr. 1982;36(5):936-42.

34. Simões A. Reprodutibilidade e validade do questionário de atividade física habitual de Baecke modificado em idosos saudáveis [dissertação]. São Paulo: Universidade Nove de Julho; 2009.

35. Finney DJ. Statistics for biologists. London: Chapman and Hall; 1980.

36. Trueblood PR, Hodson-Chennault N, McCubbin A, Youngclarke D. Performance and impairment-based assessments among community dwelling elderly: sensitivity and specificity. J geriatr phys ther. 2001;24(1):2-6.

37. Dai B, Ware WB, Giuliani CA. A structural equation model relating physical function pain impaired mobility (IM) and falls in older adults. Arch Gerontol Geriatr. 2012;55(3):645-52.

38. Greene BR, O'donovan A, Romero-Ortuno R, Cogan L, Scanaill CN, Kenny RA. Quantitative falls risk assessment using the timed up and go test. IEEE Trans Biomed Eng. 2010;57(12):2918-26.

39. Greene BR, Doheny EP, Walsh C, Cunningham C, Crosby L, Kenny RA. Evaluation of falls risk in communitydwelling older adults using body-worn sensors. Gerontology. 2012;58(5):472-480.

40. Jalali MM, Gerami H, Heidarzadeh A, Soleimani R. Balance performance in older adults and its relationship with falling. Aging Clin Exp Res. 2015;27(3):287-96.

41. Bongue B, Dupré C, Beauchet O, Rossat A, Fantino B, Colvez A. A screening tool with five risk factors was developed for fall-risk prediction in community-dwelling elderly. J Clin Epidemiol. 2011;64(10):1152-60.

42. Muir SW, Berg K, Chesworth B, Klar N, Speechley M. Balance impairment as a risk factor for falls in communitydwelling older adults who are high functioning: a prospective study. Phys Ther. 2010;90(3):338-47.

43. Gai J, Gomes L, Nóbrega OT, Rodrigues MP. Factors related to falls of elderly women residents in a community. Rev 
Assoc Med Bras. 2010;56(3):327-32.

44. Rose DJ, Lucchese N, Wiersma LD. Development of a multidimensional balance scale for use with functionally independent older adults. Arch Phys Med Rehabil. 2006;87(11):1478-85.

45. Uusi-Rasi K, Kannus P, Karinkanta S, Pasanen M, Patil R, Lamberg-Allardt C, et al. Study protocol for prevention of falls: A randomized controlled trial of effects of vitamin D and exercise on falls prevention. BMC Geriatr. 2012;12:12. doi: 10.1186/1471-2318-12-12.

46. Pepe MS. Receiver operating characteristic methodology. J Am Stat Assoc. 2000;95:308-11.

47. Vassallo M, Poynter L, Sharma JC, Kwan J, Allen SC. Fall risk-assessment tools compared with clinical judgment: an evaluation in a rehabilitation ward. Age Ageing. 2008;37(3):277-81.

48. Scott V, Votova K, Scanlan A, Close J. Multifactorial and functional mobility assessment tools for fall risk among older adults in community home-support long-term and acute care settings. Age Ageing. 2007;36:130-139.

49. Nunes CHSS, Primi R. Aspectos técnicos e conceituais da ficha de avaliação dos testes psicológicos. Em Conselho Federal de Psicologia (CFP) Avaliação Psicológica: diretrizes na regulamentação da profissão. Brasília: CFP; 2010.

50. Said AM, Manaf H, Bukry SA, Justine M. Mobility and Balance and Their Correlation with Physiological Factors in Elderly with Different Foot Postures. Biomed Res Int. 2015;2015:385269.

51. Singh DK, Pillai SG, Tan ST, Tai CC, Shahar S. Association between physiological falls risk and physical performance tests among community-dwelling older adults. Clin Interv Aging. 2015;13(10):1319-26.

52. Dong X, Chang ES, Simon MA. Physical function assessment in a community-dwelling population of U.S. Chinese older adults. J Gerontol A Biol Sci Med Sci. 2014;69(S2):S31-8.

53. Perracini MR, Ramos LR. Fatores associados a quedas em uma coorte de idosos residentes na comunidade. Rev Saude Publica. 2002;36(6):709-16.

ENDEREÇO DE CORRESPONDÊNCIA:

Ana Carolina Silva de Souza Moreira

Rua Pascoal Simone, 358

Florianópolis - SC- BRASIL

CEP: $88080-350$

E-mail: anakarolfisio@gmail.com

Aceito: 28/09/2017 